\title{
Hyper-Rayleigh scattering studies of silver, copper, and platinum nanoparticle suspensions
}

\author{
Robert C. Johnson, Jiangtian Li, Joseph T. Hupp *, George C. Schatz ${ }^{1}$ \\ Department of Chemistry, Materials Research Center, and Center for Nanofabrication and Molecular Self-Assembly, \\ 2145 Sheridan Road, Northwestern University, Evanston, IL 60208-3113, USA
}

Received 23 January 2002; in final form 5 March 2002

\begin{abstract}
The optical frequency doubling properties of silver, copper, and platinum nanoparticles in aqueous solution are studied via hyper-Rayleigh scattering (HRS) spectroscopy. Substantial HRS responses are observed for silver and copper particles. The response is attributed to enhancement due to resonance of the nonlinearly scattered light with the particles' surface plasmon absorption band. Platinum particles, which lack visible-region plasmon absorption, do not display detectable HRS signals. The largest signals from silver particles are observed under conditions of two-photon resonance, qualitatively consistent with the predictions of available theory; smaller yet still impressive signals are observed under pre- and post-resonant conditions. (c) 2002 Published by Elsevier Science B.V.
\end{abstract}

\section{Introduction}

Nanometer-sized colloidal particles of freeelectron metals display interesting and useful optical properties. Their linear optical response is dominated by the surface plasmon resonance associated with the collective oscillation of the particles' free electrons (conduction band electrons). While the linear optical properties of metal nanoparticles have been extensively investigated, the potential of these materials for second-order nonlinear optical applications - including second-

\footnotetext{
${ }^{*}$ Corresponding author. Fax: +1-847-491-7713.

E-mail addresses: jthupp@chem.nwu.edu (J.T. Hupp), schatz@chem.nwu.edu (G.C. Schatz).

${ }^{1}$ Also corresponding author.
}

order photonics applications ('hyperphotonics') has been relatively untapped. Second-order molecular nonlinear optical behavior is commonly quantified by reporting components of the first hyperpolarizability tensor $\beta$. Hyper-Rayleigh scattering (HRS), or incoherent second harmonic generation (SHG), has emerged over the last decade as a powerful technique to measure $\beta$ values of species in solution [1]. In isotropic media, conventional coherent SHG is forbidden in the electric dipole approximation [2]. HRS works, despite the orientational randomization, because signals scale as the variance of the orientation of the species in solution with respect to the incident radiation field [3]. Furthermore, HRS provides advantages over the more standard technique for measuring $\beta$, electric field-induced second harmonic generation (EFISHG). In an EFISHG experiment, sample 
anisotropy (necessary for bulk frequency doubling) is induced by poling the sample in an electric field, conditions unsuitable for analysis of charged or non-dipolar species. HRS is not limited by these restrictions and in fact has been used to investigate ionic and non-dipolar molecules [4-6].

Here we report on HRS studies of silver, copper, and platinum nanoparticle suspensions. This work follows several recent reports of substantial HRS from nanocrystalline gold particle suspensions [7-10] and reports of SHG from highly concentrated gold nanoparticle samples [11,12]. HRS signals from gold nanoparticles were observed to exceed by factors of up to $10^{5}$ those observed from suspensions of similarly sized wide bandgap semiconductor particles [13] and silicondioxide insulator particles [14]. The SHG study showed conclusively, via excitation profile measurements, that the nonlinear response is dramatically enhanced by resonance interactions with the gold plasmon band. There also exist several reports of HRS from silver nanoparticles - possibly the earliest reports of HRS from any nanoparticle [15-17].

In this Letter, we report that silver nanoparticles are exceptionally strong hyper-Rayleigh scatterers and that the scattering is clearly plasmon enhanced. We report similarly strong HRS from copper nanoparticles - another free-electron metal - under two-photon plasmon resonance conditions, but an absence of detectable HRS from platinum nanoparticles. The key difference is the absence of the visible-region plasmon band, necessary for resonance enhancement.

For macroscopic samples/interfaces, coherent SHG has proven to be sensitive to metal-based plasmon resonance $[18,19]$. We reinforce here that HRS is an effective probe of nonlinear optical behavior on an interface size regime essentially inaccessible by coherent SHG.

\section{Experimental}

Aqueous colloidal solutions of spherical polymer-stabilized silver and copper nanoparticles were synthesized following literature procedures $[20,21]$. The average particle diameters were 20 and
$12 \mathrm{~nm}$, respectively. The platinum colloid was prepared using a literature method that generates a mixture of cubic and tetrahedral particles averaging 8-10 $\mathrm{nm}$ in diameter [22].

HRS measurements were performed by using a previously described setup [7]. Briefly, the output from a mode-locked Ti:sapphire laser $(82 \mathrm{MHz}, 80$ fs pulses, $1.0 \mathrm{~W}$ average power at $820 \mathrm{~nm}$ ) was focused into a solution of nanoparticles. The scattered light (collected over a wide angle centered at $90^{\circ}$ ) was collimated and focused onto a photomultiplier tube for detection. Appropriate optical filters were used to ensure detection of only the frequency-doubled light. The signal was retrieved with a digital lock-in amplifier and recorded via computer. A small portion of the incident beam was directed through a doubling crystal and onto a fast photodiode; this signal was collected simultaneously with the scattering to account for fluctuations in laser power and modelocking stability during the experiments. HRS experiments at $1064 \mathrm{~nm}$ were performed using a $\mathrm{Nd}$ :YAG laser operating at $10 \mathrm{~Hz}(300 \mathrm{ps}, 40 \mathrm{~mJ}$ pulses) and a detection setup similar to that described by Clays and Persoons [3]. Gated electronics and boxcar integration were used to ensure detection of the HRS signal resulting from every pulse.

\section{Results and discussion}

\subsection{Measurement and analysis}

For a two-component (solvent and analyte) solution, the HRS intensity $I_{2 \omega}$ is given by

$I_{2 \omega}=G\left\langle N_{\mathrm{s}} \beta_{\mathrm{s}}^{2}+N_{\mathrm{a}} \beta_{\mathrm{a}}^{2}\right\rangle I_{\omega}^{2} \times 10^{-N_{\mathrm{a}} \varepsilon l}$,

where $I_{\omega}$ is the incident light intensity; $G$ is a parameter reflecting instrumental factors, collection efficiency, and local field factors; $s$ and $a$ denote solvent and analyte, respectively; $N$ represents concentration; $\varepsilon$ is the molar absorptivity of the solute at the second harmonic wavelength; $l$ is a pathlength term; and the brackets indicate an orientational average. The exponential term accounts for self-absorption of the HRS light by the analyte. Persoons and co-workers have empha- 
sized that, for solvents of known hyperpolarizability, Eq. (1) implies the use of solvent scattering as an internal standard.

While the significance of $\beta$ for a molecular chromophore is unambiguous, some clarification is necessary for hyperpolarizability measurements, or comparisons, involving nanoparticles. For photonic applications, perhaps the best figure of merit is $\beta^{2}$ /unit volume, since signals scale as $\beta^{2}$, not $\beta$. An alternative, and somewhat more conveniently calculated, figure of merit - though one that admittedly neglects packing differences, bondlength differences, etc., between various kinds of chromophoric materials - is $\beta_{\text {particle }}^{2} /$ atom. We present our data in this fashion. Conventional hyperpolarizability units are retrieved by reporting the square root of this quantity, $\left(\beta_{\text {particle }}^{2} / \text { atom }\right)^{0.5}$, which we label $\beta^{\prime}$. The distinction between $\beta$, $\beta /$ atom and $\beta^{\prime}$ is unimportant for comparisons involving similarly sized chromophores. However, a single nanoparticle contains tens or hundreds of thousands of atoms while most molecular chromophores contain less than a hundred; similarly, for example, a 100-nm particle contains 8000 times the number of atoms found in a $5-\mathrm{nm}$ particle of the same material. Clearly, in these cases, only normalized comparisons are informative, with $\beta^{\prime}\left(=\beta_{\text {particle }} / \sqrt{ }\right.$ atom $)$ representing a more fundamentally meaningful comparison than $\beta_{\text {particle }} /$ atom.

\subsection{Linear spectral behavior}

Fig. 1 compares experimental linear extinction spectra for each of four metal nanoparticles, measured as colloidal suspensions in water. Mie theory results for $\mathrm{Ag}$ (diameter $=20 \mathrm{~nm}), \mathrm{Au}(12$ $\mathrm{nm}), \mathrm{Cu}(12 \mathrm{~nm})$, and $\mathrm{Pt}(8 \mathrm{~nm})$ are shown in Fig. 2 for comparison. The dielectric constants for the Mie calculations were taken from Palik [23], and were further corrected for surface scattering effects using standard expressions [24].

Fig. 1 shows that the measured extinction spectra for $\mathrm{Ag}, \mathrm{Au}$, and $\mathrm{Cu}$ exhibit plasmon absorption bands with maxima at 410, 520, and 564 $\mathrm{nm}$, respectively. Pt nanoparticles, on the other hand, yield a featureless spectrum. The comparison with theory is excellent. For these relatively

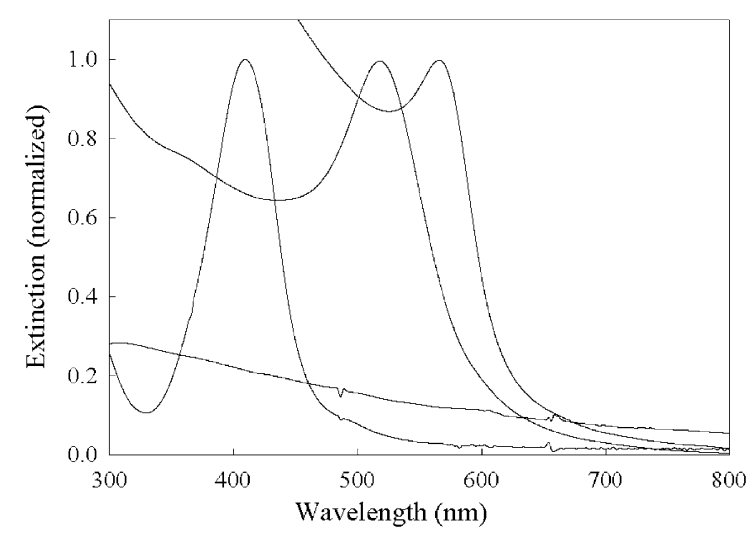

Fig. 1. Experimental extinction spectra of aqueous solutions of the metal nanoparticles studied here. The spectra with sharp surface plasmon absorption peaks are, from left to right, silver, gold, and copper nanoparticles; the featureless spectrum is of platinum nanoparticles.

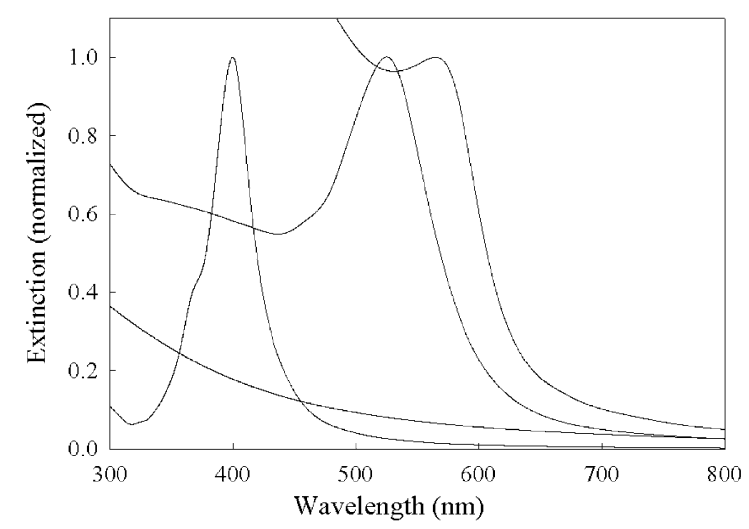

Fig. 2. Calculated extinction spectra of the metal nanoparticles studied here. Note the good agreement with experiment (Fig. 1).

small particles, the extinction cross section $C_{\text {ext }}$, is approximately proportional to $\operatorname{Im}\left\{\left[\left(\varepsilon_{\mathrm{m}} / \varepsilon_{\mathrm{s}}\right)-1\right] /\right.$ $\left.\left[\left(\varepsilon_{\mathrm{m}} / \varepsilon_{\mathrm{s}}\right)+2\right]\right\}$, where $\varepsilon_{\mathrm{m}}$ is the frequency-dependent dielectric constant of the metal nanoparticle and $\varepsilon_{\mathrm{s}}$ is the dielectric constant of the surrounding medium [25]. The plasmon resonance in the linear extinction spectrum therefore occurs at wavelengths for which $\operatorname{Re}\left(\varepsilon_{\mathrm{m}}\right) \approx-2 \varepsilon_{\mathrm{s}}$, and the resonance width is determined by the imaginary part of $\varepsilon_{\mathrm{m}} / \varepsilon_{\mathrm{s}}$. Note that $\operatorname{Im}\left(\varepsilon_{\mathrm{m}}\right)$ is large on the blue side of the plasmon resonance for $\mathrm{Cu}$ and $\mathrm{Au}$ due to interband transitions and as a result the extinction 
remains large. The resonance condition for $\mathrm{Pt}$ is satisfied only for wavelengths to the blue of those considered in Fig. 1 (i.e., at $275 \mathrm{~nm}$ ), and since $\operatorname{Im}\left(\varepsilon_{\mathrm{m}}\right)$ is especially large for $\mathrm{Pt}$, the resonance is extremely broad and hardly distinguishable.

\subsection{Nonlinear spectral behavior}

It is important to establish that signals observed at $2 \omega$ are due only to HRS. Measurements on 20$\mathrm{nm}$ colloidal silver suspensions covering a range of concentrations and incident light intensities showed quadratic dependence of the signal on the incident intensity, as expected (Eq. (1)) for a second-order process; see, for example, Fig. 3. Also observed was a linear dependence of the signal on silver atom concentration (after correction for selfabsorption). Residual coherent SHG, if present, would yield a quadratic dependence on analyte concentration [26]. Further measurements entailing detection over a range of wavelengths including the second harmonic showed the nonlinear response from each sample to be nearly monochromatic, as shown, for example, in Fig. 4. This indicates that processes such as multi-photon-absorption-induced emission (which would appear as a broad background signal under the sharp HRS response) do not contribute to the signals.

Using water as an internal standard $\left(\beta_{\text {water }}=\right.$ $0.56 \times 10^{-30}$ esu [27]), Eq. (1) yields a $\beta_{\text {silver }}^{\prime}$ value

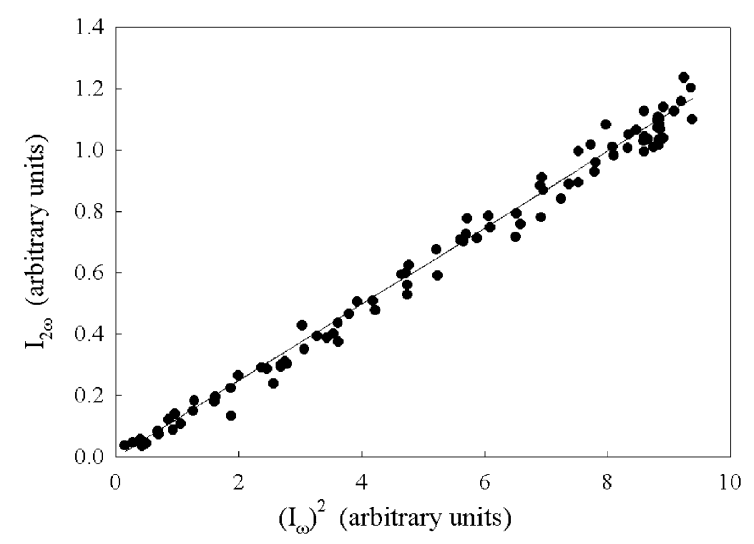

Fig. 3. Dependence of the observed frequency-doubled signal, $I_{2 \omega}$, on the square of the incident laser intensity, $\left(I_{\omega}\right)^{2}$ for a solution of silver nanoparticles. The quadratic power dependence is indicative of HRS.

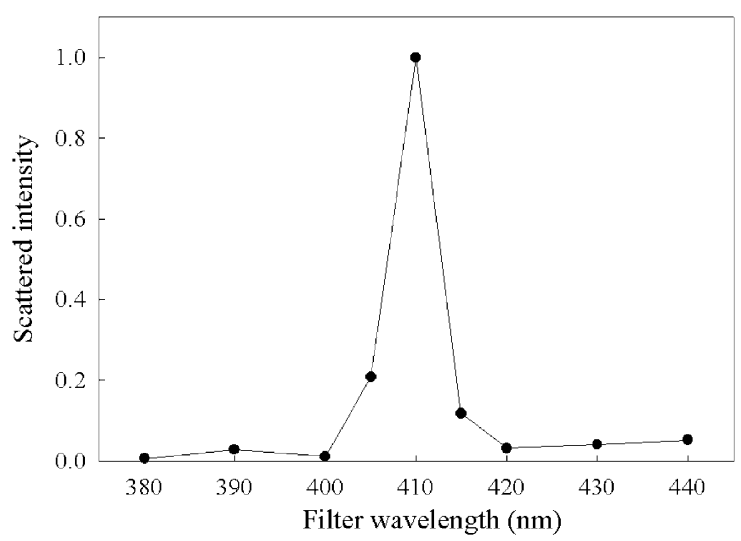

Fig. 4. Scattered intensity as a function of detected wavelength (notch filter) for a solution of silver nanoparticles. The nearly monochromatic response is characteristic of HRS.

of $5600( \pm 1100) \times 10^{-30}$ esu at an excitation wavelength of $820 \mathrm{~nm}$. As shown in Fig. 5, the hyperpolarizability varies as a function of the incident wavelength, yielding smaller but still impressive $\beta_{\text {silver }}^{\prime}$ values above and below $820 \mathrm{~nm}$. In this region, the incident radiation is nonresonant with the plasmon absorption. The nonlinearly scattered radiation at $2 \omega$, on the other hand, is resonant with the plasmon feature $\left(\lambda_{\max }=410 \mathrm{~nm}\right.$; Figs. 1 and 2).

Similar experiments on aqueous suspensions of 12-nm diameter copper nanoparticles yielded a

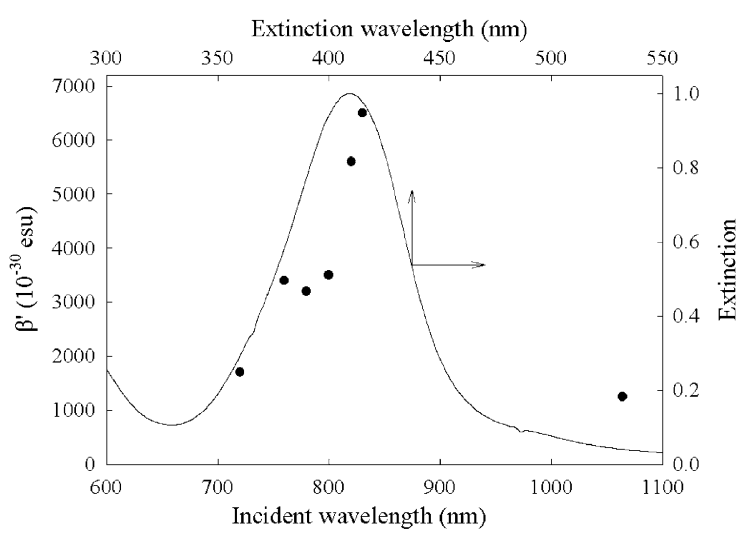

Fig. 5. Hyperpolarizability of colloidal silver particles as a function of incident wavelength (circles) and the particles' extinction spectrum (line). The trend in the hyperpolarizability response indicates two-photon resonant enhancement. 
$\beta_{\text {copper }}^{\prime}$ value of $1600( \pm 120) \times 10^{-30}$ esu at an excitation wavelength of $820 \mathrm{~nm}$. In contrast, similarly sized tetrahedral platinum particles yielded no detectable HRS. Recalling that $\beta_{\text {gold }}^{\prime}$ is similarly large $\left(\sim 2800 \times 10^{-30}\right.$ esu for incident light at 820 $\mathrm{nm}$ [7]), it seems clear that the key difference between platinum and the coinage metals is the absence of a visible-region plasmon band. (The values reported in [7] are incorrectly labeled $\beta /$ atom; they are, in fact, $\beta^{\prime}$ values. The seemingly much smaller values reported in [10], on the other hand, are values of $\beta /$ atom. When converted to $\beta^{\prime}$, they are in reasonable agreement with those in [7].)

Returning to the excitation profile for silver, available theory by Agarwal and Jha [28] supports the notion that 2-photon plasmon-resonance enhancement effects should exist. (More recent theoretical studies by Hua and Gersten [29], Bennemann and co-workers [30], and Dadap and co-workers [31] also predict 2-photon resonance enhancement.) In the Agarwal-Jha work, the power radiated at $2 \omega$ and integrated over all solid angles is found to be

$$
\begin{aligned}
S= & 192 \pi^{2} c|E(\omega)|^{4}\left(\frac{2 \omega R}{c}\right)^{6} \\
& \times\left\{\left|\frac{e[\varepsilon(2 \omega)-1] / 8 \pi m \omega^{2}}{[\varepsilon(\omega)+2][\varepsilon(2 \omega)+2]}\right|^{2}\right. \\
& \left.+\frac{36}{5}\left|\frac{e[\varepsilon(\omega)-1] / 8 \pi m \omega^{2}}{[\varepsilon(\omega)+2]^{2}[2 \varepsilon(2 \omega)+3]}\right|^{2}\right\},
\end{aligned}
$$

where $e$ and $m$ are the electron charge and effective mass, $E(\omega)$ is the field at the fundamental frequency, $R$ is the sphere radius, $c$ is the velocity of light, $\varepsilon(\omega)$ is the relative dielectric constant (defined as $\left.\varepsilon_{\mathrm{m}} / \varepsilon_{\mathrm{s}}\right)$ at incident radiation frequency $(\omega)$, and $\varepsilon(2 \omega)$ is the corresponding quantity at $2 \omega$ Notice that the first term in the large brackets has the characteristic dipole plasmon resonance denominator $[\varepsilon(\omega)+2]^{-1}$. Strong enhancement of the HRS signal is expected, therefore, at incident wavelengths coincident with the dipole plasmon absorption maximum. Additionally, the term containing the factor $[\varepsilon(2 \omega)+2]^{-2}$ indicates strong HRS enhancement at an incident wavelength that is double the dipole plasmon absorption wave- length, and the term containing $[2 \varepsilon(2 \omega)+3]^{-2}$ gives strong HRS at double the quadrupole plasmon wavelength. For wavelengths close to $820 \mathrm{~nm}$, the dipole resonance would be dominant for Ag, and indeed this effect is clearly evident in the experiments summarized in Fig. 5. Note that the expression for $\mathrm{S}$ indicates a dependence on the sixth power of the particle radius. This means that the intensity per atom is predicted to vary as the cube of the radius, which is clearly an important factor if one is comparing particles of different size.

We have used the Argarwal-Jha theory to predict HRS intensities (per atom) at $820 \mathrm{~nm}$, using dielectric constants for $\mathrm{Ag}, \mathrm{Au}, \mathrm{Cu}$, and $\mathrm{Pt}$ from Palik (and corrected for surface scattering), and with the same sphere radii that were used in the extinction calculation. After normalizing the result to unity for $\mathrm{Cu}$, we obtain the intensities per atom of $80,0.7,1.0$, and 0.1 for $\mathrm{Ag}, \mathrm{Au}, \mathrm{Cu}$, and $\mathrm{Pt}$. These results indicate that silver should have a much larger HRS signal at $820 \mathrm{~nm}$ than the other metals, with $\mathrm{Au}$ and $\mathrm{Cu}$ being about the same, and Pt significantly smaller. Although the difference between $\mathrm{Ag}$ and the other metals is not found in the measurements, the other conclusions match the observations reasonably. The theory calculation indicates that the $\mathrm{Ag}$ intensity is especially large because the dipole plasmon resonance peak occurs at this wavelength. Away from resonance, say at $1000 \mathrm{~nm}$, the Ag intensity is predicted to be down by a factor of 20 .

The Argarwal-Jha theory makes a number of assumptions that hinder quantitative comparisons with experiment, including the use of free electron expressions for the nonlinear susceptibilities and neglect of finite wavelength corrections to the quasistatic expressions for the HRS cross-section. An alternative to this theory that avoids these problems was developed in the work of Zeman and Schatz [32]. They use the metal-dependent surface electromagnetic field that arises from plasmon excitation to define an enhancement factor for HRS. This factor is given by $\Gamma=|E(\omega)|^{4}|E(2 \omega)|^{2}$, where $|E|$ refers to the average local surface field. This expression is analogous to what has often been used in modeling surface enhanced Raman scattering enhancement factors. In the present 
application we have evaluated the fields using the quasistatic approximation, and the same dielectric constants and structural properties as in the Agarwal-Jha calculation. The normalized intensities (per atom) that we calculate are 61, 0.7, 1.0, and 0.2 for $\mathrm{Ag}, \mathrm{Au}, \mathrm{Cu}$, and $\mathrm{Pt}$, respectively. Evidently these results are very similar to the Agarwal-Jha predictions. Indeed, the results for Ag, $\mathrm{Cu}$, and $\mathrm{Au}$ are similar to what Zeman and Schatz found earlier in their modeling of the island film experiments of Chen et al. [33]. Zeman and coworkers [34] obtained reasonable correspondence with these experiments for $\mathrm{Ag}, \mathrm{Au}$, and $\mathrm{Cu}$ and a subsequent study of Pt films was also successful. However the present comparison is much better defined, as the particles have more homogenous size and shape distributions, and they are, as best as we can determine, dispersed. The theory/experiment disparities suggest that further work is needed to develop a more quantitative theory of HRS intensities.

One additional prediction of either the Agarwal-Jha theory or the Zeman-Schatz theory is that one-photon resonance, as opposed to two-photon resonance, should result in exceptionally large HRS responses. Thus for Ag, the theory predicts intensities at $410 \mathrm{~nm}$ that are nearly a factor of 10 higher than at $820 \mathrm{~nm}$. This suggests that much larger HRS signals would be obtained if the experiments were done at shorter wavelengths. However, the clear danger here is that photo damage [35] - not a concern at $820 \mathrm{~nm}$ - will dominate the experimental response.

\section{Conclusions}

The nonlinear optical responses of nanometerscale silver, copper, and platinum particles in aqueous solution were examined by hyper-Rayleigh scattering, complementing previous studies of gold and silver nanoparticles. Silver and copper particles, which possess intense visible region surface plasmon absorption bands, prove to be excellent nonlinear scatterers; platinum particles, which lack surface plasmon absorption in the visible, and for which the plasmon is strongly damped, yield no detectable nonlinear optical signal. For silver nanoparticles, the most efficient nonlinear optical response is obtained when the second harmonic of the incident light coincides with the particles' plasmon absorption maximum. This result agrees with the predictions of electromagnetic enhancement calculations, however the theory predicts a much greater difference between silver and either gold or copper than is seen in the experiments.

\section{Acknowledgements}

We thank the Army Research Office (MURI program) and the Northwestern Materials Research Center (NSF-MRSEC program) for support of our work. We also thank Professor Ken Spears for the use of the Nd:YAG laser.

\section{References}

[1] E. Hendrickx, K. Clays, A. Persoons, Acc. Chem. Res. 31 (1998) 675.

[2] J.A. Giordmaine, Phys. Rev. 138 (1965) A1599.

[3] K. Clays, A. Persoons, Rev. Sci. Instrum. 63 (1992) 3285.

[4] B.J. Coe, M.C. Chamberlain, J.P. Essex-Lopresti, S. Gaines, J.C. Jeffery, S. Houbrechts, A. Persoons, Inorg. Chem. 36 (1997) 3284.

[5] F.W. Vance, J.T. Hupp, J. Am. Chem. Soc. 121 (1999) 4047.

[6] C. Dhenault, I. Ledoux, I.F.W. Samuel, J. Zyss, M. Bourgault, H. Le Bozec, Nature 374 (1995) 339.

[7] F.W. Vance, B.I. Lemon, J.T. Hupp, J. Phys. Chem. B 102 (1998) 10091.

[8] R.C. Johnson, J.T. Hupp, in: D.L. Feldheim, C. Foss (Eds.), Metal Nanoparticles: Synthesis, Characterization, and Applications, Marcel-Dekker, New York, 2002.

[9] P. Galletto, P.F. Brevet, H.H. Girault, R. Antoine, M. Broyer, J. Phys. Chem. B 103 (1999) 8706.

[10] P. Galletto, P.F. Brevet, H.H. Girault, R. Antoine, M. Broyer, Chem. Commun. (1999) 581.

[11] R. Antoine, M. Pellarin, B. Palpant, M. Broyer, B. Prével, P. Galletto, P.F. Brevet, H.H. Girault, J. Appl. Phys. 84 (1998) 4532

[12] R. Antoine, P.F. Brevet, H.H. Girault, D. Bethell, D.J. Schiffrin, Chem. Commun. (1997) 1901.

[13] B.I. Lemon, Ph.D. Thesis, Northwestern University, 1999.

[14] F.W. Vance, B.I. Lemon, J.A. Ekhoff, J.T. Hupp, J. Phys. Chem. B 102 (1998) 1845.

[15] C.K. Johnson, J.A. Soper, J. Phys. Chem. 93 (1989) 7281.

[16] K. Clays, E. Hendrickx, M. Triest, A. Persoons, J. Mol. Liq. 67 (1995) 133. 
[17] A.V. Baranov, Ya.S. Bobovich, V.I. Petrov, Opt. Spectrosc. 61 (1986) 315.

[18] M.L. Sandrock, C.D. Pibel, F.M. Geiger, C.A. Foss, J. Phys. Chem. B 103 (1999) 2668.

[19] R. Srinivasan, I.I. Suni, J. Electrochem. Soc. 146 (1999) 570.

[20] E.R. Savinova, A.L. Chuvilin, V.N. Parmon, J. Mol. Cat. 48 (1988) 217

[21] P.C. Lee, D. Meisel, J. Phys. Chem. 86 (1982) 3391.

[22] T.S. Ahmadi, Z.L. Wang, T.C. Green, A. Henglein, M.A. El-Sayed, Science 272 (1996) 1924.

[23] E.W. Palik, Handbook of Optical Constants, Academic Press, New York, 1985.

[24] W.A. Kraus, G.C. Schatz, Chem. Phys. Lett. 99 (1983) 353.

[25] C.M. Sorenson, in: K.S. Birdi (Ed.), Handbook of Surface and Colloid Chemistry, CRC Press, Boca Raton, FL, 1997, p. 537.
[26] R.A. Corn, D.A. Higgins, Chem. Rev. 94 (1994) 107.

[27] C. Boutton, K. Clays, Personal communication.

[28] G.S. Agarwal, S.S. Jha, Solid State Commun. 41 (1982) 499.

[29] X.M. Hua, J.I. Gersten, Phys. Rev. B 33 (1986) 3756.

[30] D. Ostling, P. Stampfli, K.H. Bennemann, Z. Physik D 28 (1993) 169.

[31] J.I. Dadap, J. Shan, K.B. Eisenthal, T.F. Heinz, Phys. Rev. Lett. 83 (1999) 4045.

[32] E.J. Zeman, G.C. Schatz, J. Phys. Chem. 91 (1987) 634.

[33] C.K. Chen, A.R.B. de Castro, Y.R. Shen, Phys. Rev. Lett. 46 (1981) 145.

[34] K.L. Haller, L.A. Bumm, R.I. Altkorn, E.J. Zeman, G.C. Schatz, R.P. Van Duyne, J. Chem. Phys. 90 (1989) 1237.

[35] N. Chandrasekharan, P.V. Kamat, J.Q. Hu, G. Jones, J. Phys. Chem. B 104 (2000) 11103. 